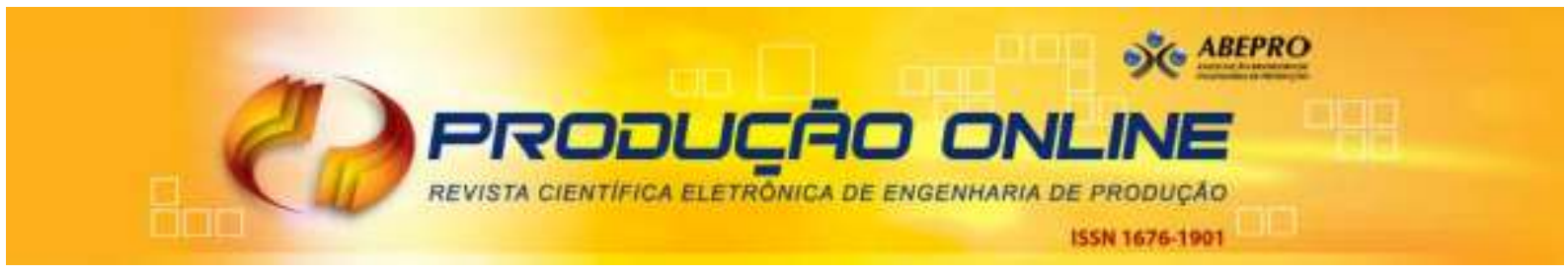

\title{
ANÁLISE DA PRÁTICA VENDOR MANAGED INVENTORY (VMI) EM UM FORNECEDOR DE BENS DE CONSUMO
}

\section{VENDOR MANAGED INVENTORY (VMI) PRACTICE ANALYSIS IN A SUPPLIER OF CONSUMER GOODS}

\author{
Denise Cervilha de Freitas* E-mail: decervilha@hotmail.com \\ Robson Nogueira Tomas* E-mail: rn.tomas@uol.com.br \\ Rosane Lúcia Chicarelli Alcântara* E-mail: rosane@dep.ufscar.br \\ Universidade Federal de São Carlos (UFSCar), São Carlos, SP
}

\begin{abstract}
Resumo: Uma das práticas colaborativas mais discutidas na literatura é o Vendor Managed Inventory (VMI), que dá ao fornecedor a liberdade de tomar as decisões de reabastecimento de seu cliente, permitindo o melhor planejamento de sua produção. Apesar dos esforços de vários pesquisadores, poucos estudos confrontam a realidade enfrentada pelas empresas com as teorias presentes na literatura. Sendo assim, o presente artigo tem como objetivo a análise da prática do VMI de um fornecedor de bens de consumo com um de seus clientes varejistas, a partir das barreiras que limitam os potenciais resultados da prática e dos fatores críticos para o sucesso do VMI. Baseado nesta análise, este artigo também apresenta algumas proposições para estudos futuros. A revisão bibliográfica permitiu a identificação dos elementos que influenciam na adoção e manutenção do VMI, que foram agrupados em elementos comportamentais, culturais e físicos. O método utilizado foi o estudo de caso com entrevistas em profundidade, que permitiu identificar os elementos que limitam os potenciais ganhos do VMI na empresa analisada e também aqueles que contribuem para o sucesso da prática.
\end{abstract}

Palavras-chave: VMI. Barreiras. Fatores Críticos. Empresas brasileiras. Cadeia de Suprimentos.

Abstract: One of the widely discussed collaborative practices in the literature is Vendor Managed Inventory (VMI), which gives supplier the freedom to take decisions regarding customer's replenishment, allowing better production planning. Despite the efforts of many researches, few studies compare the reality faced by companies with theories in the literature. Thus, this research aims to analyze the VMI practice of a consumer goods supplier with its retail customer, based on the barriers that limit the potential results of the practice and also the critical factors for the success of VMI. Insights from this analysis resulted in some propositions for future studies. The literature review allowed the identification of elements that influence the adoption and maintenance of VMI that were grouped into behavioral, cultural and physical elements. The method used was a case study with indepth interviews, which allowed identifying the elements that limit the potential gains of VMI in the analyzed company and also those ones that contribute to the success of the practice.

Keywords: VMI. Barriers. Critical Factors. Brazilian companies. Supply Chain.

\section{INTRODUÇÃO}

O novo paradigma competitivo e gerencial revela que as empresas não competem mais isoladamente como entidades autônomas, mas cada vez mais dentro de cadeias de suprimentos (LAMBERT, 2008; PIRES, 2010). Neste sentido, Revista Produção Online, Florianópolis, SC, v.14, n. 3, p. 1024-1049, jul./set. 2014. 
Whipple e Russel (2007) afirmam que o desenvolvimento de iniciativas de colaboração na cadeia de suprimentos permite relacionamentos interorganizacionais mais fortes, com a intensificação da troca de informações, o que melhora a qualidade da tomada de decisão, reduz a incerteza da demanda e, em última instância, melhora o desempenho da cadeia de suprimentos.

Neste sentido, os mesmos autores narram que nos anos 90, uma iniciativa colaborativa chamada Efficient Consumer Response (ECR) introduziu a filosofia de compartilhamento de informações estratégicas, em face à mentalidade da época em manter as informações internamente. A adaptação e evolução desta iniciativa originaram outras abordagens colaborativas, incluindo o Vendor Managed Inventory (VMI), Continuous Replenishment (CR) e Collaborative Planning, Forecasting, and Replenishment (CPFR).

A adoção do VMI promete uma relação ganha-ganha entre o fornecedor e o cliente. Em seu cenário ideal, é dada ao fornecedor a liberdade de planejar sua própria produção e também de programar o reabastecimento de seu cliente, dado que os níveis de serviço foram anteriormente acordados (CLAASSEN; VAN WEELE; VAN RAAIJ, 2008). Waller, Johnson e Davis (1999) afirmam ainda que os custos de investimentos em tecnologia para a adoção do VMI estão ficando cada vez menos dispendiosos, contribuindo para a vasta utilização da prática entre as empresas.

O VMI é uma das iniciativas de colaboração mais discutidas para melhorar a eficiência das empresas de uma cadeia de suprimentos (WALLER; JOHNSON; DAVIS, 1999). Whipple e Russel (2007) classificam o VMI como o estágio inicial de relacionamento colaborativo, que se preocupa com problemas e tarefas no nível operacional. Para eles, esse tipo de relacionamento possibilita o aumento do compartilhamento de informações, reduz os erros e melhora a solução de problemas.

Entretanto, alguns estudos realizados em empresas de grande porte mostraram que a adoção do VMI não proporcionou os resultados esperados (BARRAT, 2004; CLAASSEN; VAN WEELE; VAN RAAIJ, 2008; SARI, 2007). Neste sentido, Sari (2007) aponta alguns fatores que limitam o potencial da prática nas empresas, como informações não acuradas, confiança limitada entre os parceiros e problemas na previsão de demanda. Complementarmente, Claassen, Van Weele e 
Van Raaij (2008) atribuem às restrições acerca dos limites mínimo e máximo de inventário as causas de insucesso da prática do VMI, uma vez que limitam as decisões de reabastecimento.

Apesar da larga adoção da prática do VMI entre as empresas e dos benefícios gerados por sua utilização, os estudos que abordam a prática são predominantemente de cunho teórico, havendo uma lacuna entre os estudos e a realidade enfrentada pelas empresas. Fugate, Sahin e Mentzer (2006) afirmam que há uma desconexão entre o que é conhecido na pesquisa acadêmica e como os gerentes aplicam os mecanismos de coordenação e o que realmente acontece na prática. Sendo assim, o presente artigo tem como objetivo a analisar a prática do VMI de um fornecedor de bens de consumo com um de seus clientes varejistas, a partir das barreiras que limitam os potenciais resultados da prática e dos fatores críticos para o sucesso do VMI. A partir desta análise, este artigo também apresenta algumas proposições para estudos futuros. Espera-se com os resultados apresentados, contribuir com o entendimento do uso do VMI e de iniciativas de colaboração da cadeia de suprimentos, e auxiliar os gestores na tomada de decisão relativa às ações de colaboração com seus parceiros comerciais.

Para isso, inicialmente foi realizado uma revisão da literatura que identificou as variáveis que influenciam no resultado do VMI, e em seguida, um estudo de caso com entrevistas em profundidade com funcionários de uma empresa fornecedora de bens de consumo, o que permitiu uma análise exploratória inicial das barreiras e fatores críticos de sucesso da prática do VMI. Este artigo é composto por sete seções, incluindo esta seção de introdução. Na seção 2 são apresentados os principais aspectos de colaboração na cadeia de suprimentos, incluindo as principais iniciativas e práticas de colaboração na cadeia de suprimentos. A seção 3 apresenta o conceito do VMI, mostrando suas principais características, resultados esperados e observações de pesquisas anteriores. A seção 4, baseada nas seções anteriores apresenta os principais elementos que influenciam no VMI. A metodologia utilizada é descrita na seção 5 e a análise do estudo de caso é apresentada na seção 6 . Finalmente, a seção 7 apresenta as considerações, contribuições e limitações do trabalho. 


\section{COLABORAÇÃO NA CADEIA DE SUPRIMENTOS}

A colaboração entre os membros da cadeia de suprimentos é o fator fundamental que determina o sucesso da SCM (BALLOU, 2007). Os benefícios trazidos pela colaboração envolvem aumento da qualidade dos produtos, redução dos custos, aumento das receitas, redução do lead time e operações mais eficientes e eficazes, por meio do aumento da flexibilidade e a redução da incerteza da demanda (SOOSAY; HYLAND; FERRER, 2008).

Diversos autores, tais como Barrat (2004), Marqui, Moura e Alcântara (2013), Min et al. (2005), Vieira, Yoshizaki e Ho (2009), discutem sobre os elementos que propiciam a colaboração na Gestão da Cadeia de Suprimentos. Neste aspecto, Min et al. (2005) propõem um modelo conceitual de colaboração na cadeia de suprimentos que mostra os antecedentes (entendidos também como requisitos à colaboração), as principais atividades colaborativas para o processo de colaboração e as consequências obtidas pela correta utilização destas atividades. Dentre os antecedentes para a colaboração estão a intensão estratégica, alinhamento interno, orientação para relações, investimentos específicos para os relacionamentos, fluxo de informações livre e comunicação intensificada e formalização. Dentre as atividades colaborativas estão a divisão de informação, planejamento conjunto, resolução de problemas conjuntos, medidas de desempenho, mobilização de recursos e habilidades. Finalmente, as consequências da colaboração na cadeia de suprimentos são eficiência, eficácia, lucratividade, reforço e expansão dos relacionamentos.

Por outro lado, Vieira, Yoshizaki e Ho (2009) agrupam os elementos de colaboração em três níveis: estratégico, táticos e interpessoais. E concluem que a colaboração no nível interpessoal contribui com maior intensidade para a colaboração, sendo representada pela confiança, interdependência, flexibilidade e reciprocidade. Os elementos táticos referem-se às ações conjuntas; compartilhamento de custos, riscos e benefícios; e compartilhamento de informações logísticas e comerciais. Para eles, o nível estratégico de colaboração afeta diretamente os elementos de integração táticos e é considerado a base para o processo colaborativo. Seus elementos são: compartilhamento de informações de 
inventário; conhecimento das dificuldades e estratégias dos parceiros; história de relacionamento e envolvimento da alta gerência.

De maneira análoga, Barrat (2004) também enfatiza a importância da confiança, reciprocidade, troca de informação, abertura e comunicação como elementos chave da cultura colaborativa. Para ele, para que colaboração aconteça, é necessário existir atividades interfuncionais, alinhamento de processos, tomada de decisão conjunta e métricas relacionadas à cadeia de suprimentos (em oposição às métricas departamentais). Por fim, para que a colaboração seja sustentável, o autor defende que deve haver comprometimento de recursos, suporte intraorganizacional (gerentes e vários departamentos), foco corporativo na cadeia de suprimentos, desenvolvimento de um plano de negócios, e uso de tecnologia compatível com o volume de dados transacionados.

Marqui, Moura e Alcântara (2013) constroem uma escada para a colaboração, na qual os degraus iniciais são a cooperação e a coordenação. Elas identificam as características e os comportamentos dos relacionamentos colaborativos em cada nível da escada. Dentre as características citadas por elas, estão: comunicação, interdependência, transparência, flexibilidade, liderança, sincronização da tomada de decisão, comprometimento, compatibilidade de cultura organizacional e confiança. Entre os comportamentos estão: compartilhamento de informações, investimento em tecnologia da informação, planejamento conjunto, documentação e padronização dos processos, suporte da alta gerência, adoção de metas e objetivos comuns, adoção de modelos de medição de desempenho padronizados, integração interfuncional, desenvolvimento e manutenção de relacionamento próximo e de longo prazo, treinamento da cadeia de suprimentos.

Práticas e iniciativas de colaboração na cadeia de suprimentos, tais como o ECR (Efficient Consumer Response), VMI (Vendor Managed Inventory), QR (Quick Response), CR (Continuous Replenishment), CPFR (Collaborative Planning, Forecasting and Replenishment); também chamadas de programas de reposição automáticas (DAUGHERTY; MYERS; AUTRY, 1999; FERREIRA; ALCANTARA, 2011; PIRES, 2010) são frequentemente citadas na literatura. Williams e Tokar (2008) afirmam que as abordagens CR, ECR, VMI e QR são desenhadas para sincronizar a demanda com a oferta através do controle de inventário, por meio da 
colaboração externa. Segundo eles, o $\mathrm{CR}$ e $\mathrm{O}$ VMI são programas de reabastecimento similares, sendo que, no VMI, a decisão de reabastecimento é atribuída ao fornecedor. Além disto, os autores afirmam que o ECR está relacionado com as indústrias de grocery (gênero alimentício), enquanto o QR está voltado para a indústria de vestuário.

Ainda não existe uma unanimidade na literatura a respeito da originalidade de cada uma das iniciativas de colaboração. Alguns autores apontam que nomes diferentes são utilizados para uma mesma abordagem; outros afirmam que cada prática representa uma adaptação para um determinado ambiente; e outros ainda afirmam haver uma escala evolutiva entre elas (DISNEY; TOWILL, 2003; SARI, 2007; WALLER; JOHNSON; DAVIS, 1999; WHIPPLE; RUSSEL, 2007; WILLIAMS; TOKAR, 2008). Disney e Towill (2003) apontam nomes similares para o VMI: QR, SCR (Syncronised Consumer Response), CR, ECR, QR e CPFR; e acreditam que a terminologia utilizada depende do setor de aplicação, questões de propriedade e escopo de implantação, porém, segundo eles, todos visam a redução do efeito chicote. Para Sari (2007) e Waller, Johnson e Davis (1999), VMI e CR são a mesma prática. Por outro lado, Whipple e Russel (2007), falam sobre o surgimento do ECR e sua evolução em VMI, CR e CPFR respectivamente e, de maneira parecida, Attaran e Attaran (2007) apresenta o VMI como um tipo de relacionamento primário e o CR, ECR e CPFR como suas evoluções.

Whipple e Russel (2007) examinaram várias formas de relacionamentos colaborativos e apresentaram uma tipologia de abordagens colaborativas, composta por três diferentes estágios de colaboração: gestão de transações colaborativas, gestão de eventos colaborativos e gestão de processos colaborativos. Segundo eles, o VMI representa o primeiro tipo de colaboração, com foco na troca de dados e alinhamento de tarefas para resolução de problemas e/ou erros nas tarefas operacionais, cujo horizonte de planejamento é de curto prazo. Na gestão de eventos colaborativos, o foco passa a ser nos processos de planejamento e tomada de decisão conjuntas, associadas com eventos e/ou questões específicas. A preocupação passa a ser a eficiência da cadeia de suprimentos, evitando as rupturas e o horizonte de planejamento é de médio prazo, visando resolver problemas que irão criar impacto incremental no nível da loja. Por fim, na gestão de 
processos colaborativos o foco é na solução conjunta de problemas, planejamento de negócio a longo prazo e integração de processos, visando a eficiência e eficácia da cadeia de suprimentos, com planejamento a longo prazo.

Danese (2007) tenta entender o que leva as empresas a implantar diferentes tipos de colaboração CPFR, e, coerentemente com Whipple e Russel (2007), aponta três níveis de profundidade de colaboração: comunicação, colaboração limitada e colaboração plena. Para ela, na comunicação, as empresas apenas trocam dados e informações com o parceiro comercial; na colaboração limitada, os planos são sincronizados entre os parceiros e as exceções gerenciadas; e na colaboração plena, há a sincronização e coordenação dos planos de negócios, vendas e previsão de pedidos. Sua análise dos casos estudados mostrou que quando a cadeia de suprimentos visa a eficiência (redução de custos), uma colaboração focada apenas na comunicação de dados é suficiente; porém, quando a cadeia de suprimentos necessita ser mais responsiva, uma colaboração mais profunda é necessária.

\section{VENDOR MANAGED INVENTORY (VMI)}

Disney e Towill (2003) afirmam que Magee (1958) realizou a primeira discussão sobre a prática que hoje é conhecida como VMI. Porém, foi o sucesso da parceria entre Walmart e Procter\&Gamble na década de 80 que popularizou a iniciativa. Diversas empresas, principalmente no setor de bens de consumo duráveis implantaram o VMI, entre elas Campbell Soup, Johnson\&Johnson, Barilla (DANESE, 2006; PIRES, 2010; SARI, 2007; WALLER; JOHNSON; DAVIS, 1999). Blaterwick (1998) sugere que o termo VMI se tornou conhecido efetivamente com os projetos implantados por Walmart e K-mart, no começo da década de 90.

No VMI, as decisões de abastecimento, tais como quantidade requerida, embarques e cronogramas são feitas pelo fornecedor através do monitoramento do nível de inventário do cliente (WALLER; JOHNSON; DAVIS, 1999). Para Reddy e Vrat (2007), os sistemas de tecnologia da informação (TI) auxilia o reabastecimento dos estoques, uma vez que fornece melhores informações relacionadas à utilização, venda de produtos, inventário do cliente e outras informações relacionadas à atividade de marketing. Claassen, Van Weele e Van Raaij (2008) apontam que no VMI os níveis de serviço são acordados com o cliente e, a partir disto, o fornecedor 
pode planejar sua produção e decidir a programação dos reabastecimentos, reduzindo, ou até mesmo, eliminando os problemas de estoque de segurança.

Entre os benefícios encontrados na literatura com a adoção da prática colaborativa do VMI estão: melhoria do nível de serviço, redução do lead time, aumento do giro dos estoques, redução dos stock-outs (falta de estoque), melhoria do controle do efeito bullwhip (efeito chicote), redução dos custos (CLAASSEN; VAN WEELE; VAN RAAIJ, 1999; ELVANDER; SARPOLA; MATTSON, 2007; REDDY; VRAT, 2007), redução dos custos de introdução de novos produtos (WALLER; JOHNSON; DAVIS, 1999).

Os fornecedores se beneficiam do melhor atendimento e maior fidelização do cliente, melhor gestão da demanda e melhor conhecimento do mercado (PIRES, 2010). Waller, Johnson, Davis (1999) mostram também que o fornecedor consegue a estabilização da sua produção e a otimização dos custos de transporte. Nesse sentido, Claassen, Van Weele e Van Raaij (2008) explicam que os fornecedores conseguem alinhar seus processos produtivos com a demanda do cliente quando trabalham com o VMI. A redução da incerteza da demanda reduz a necessidade de estoques de segurança.

As empresas clientes conseguem menos custo de estocagem e menos dispêndio de capital de giro. Elas recebem melhor atendimento do fornecedor e têm as gestões de compra e inventário simplificadas (CLAASSEN; VAN WEELE; VAN RAAIJ, 2008; PIRES, 2010). Sari (2007) afirma que o aumento da disponibilidade de produtos, aumento do nível de serviço e redução dos custos de monitoramento de estoque e realização de pedido são benefícios trazidos pelo VMI.

Claassen, Van Weele, Van Raaij (2008) sugerem que a efetiva implantação do VMI envolve uma abordagem multifuncional e interorganizacional. Após revisão da literatura, eles identificaram quatro capacitadores do VMI: qualidade do relacionamento, qualidade da informação, compartilhamento de informações e qualidade dos sistemas de informação e de comunicação. Falhas nesses capacitadores resultam em implantações mal sucedidas da prática do VMI.

Adicionalmente, Pires (2010) mostra que muitas vezes o VMI é adotado equivocadamente na prática. Algumas empresas tentam aplicar o VMI simplesmente para transferir os custos de estoques aos seus fornecedores ou para se livrar da 
responsabilidade de promover um planejamento detalhado e de qualidade para gerenciar seus inventários. Danese (2006) argumenta que estes comportamentos oportunistas muitas vezes ocorrem pela presença de metas contrastantes entre os membros da cadeia de suprimentos. Vigtil (2007) defende ainda que para obter transparência e visibilidade da demanda, é fundamental que os clientes estejam dispostos a fornecerem os dados. O cliente deve ser capaz de fornecer projeções de qualidade enquanto os fornecedores devem ser capazes de suprir as demandas (BLATERWICK, 1998; VIGTIL, 2007).

Sari $(2007,2008)$ identifica alguns fatores de insucesso adoção do VMI, tais como divisão de dados não acurados ou desatualizados, falta de tecnologia de informação adequada, falta de confiança mútua. Outro ponto destacado por ele é a falha na previsão da demanda, uma vez que é feita isoladamente pelo fornecedor e não envolve os varejistas. Os estudos por ele realizados mostram que a acurácia das informações de inventário também impacta no desempenho das iniciativas de colaboração. Reddy e Vrat (2007) complementam apontando que os casos de insucesso ocorrem quando as empresas não utilizam os dados efetivamente para planejamento da produção e continuam produzindo para estocagem, quando há falta de integração dos sistemas, limitação da visibilidade de inventários e ordens. Além disto, uma barreira à implantação são os altos investimentos iniciais.

\section{ELEMENTOS QUE INFLUENCIAM O VMI}

Baseado no exposto anteriormente, os elementos que influenciam a implantação do VMI foram divididos pelos autores em elementos comportamentais, elementos culturais e elementos físicos, conforme a figura 1 a seguir, que especifica também as fontes da literatura. 
Figura 1 - Elementos que influenciam a implantação do VMI

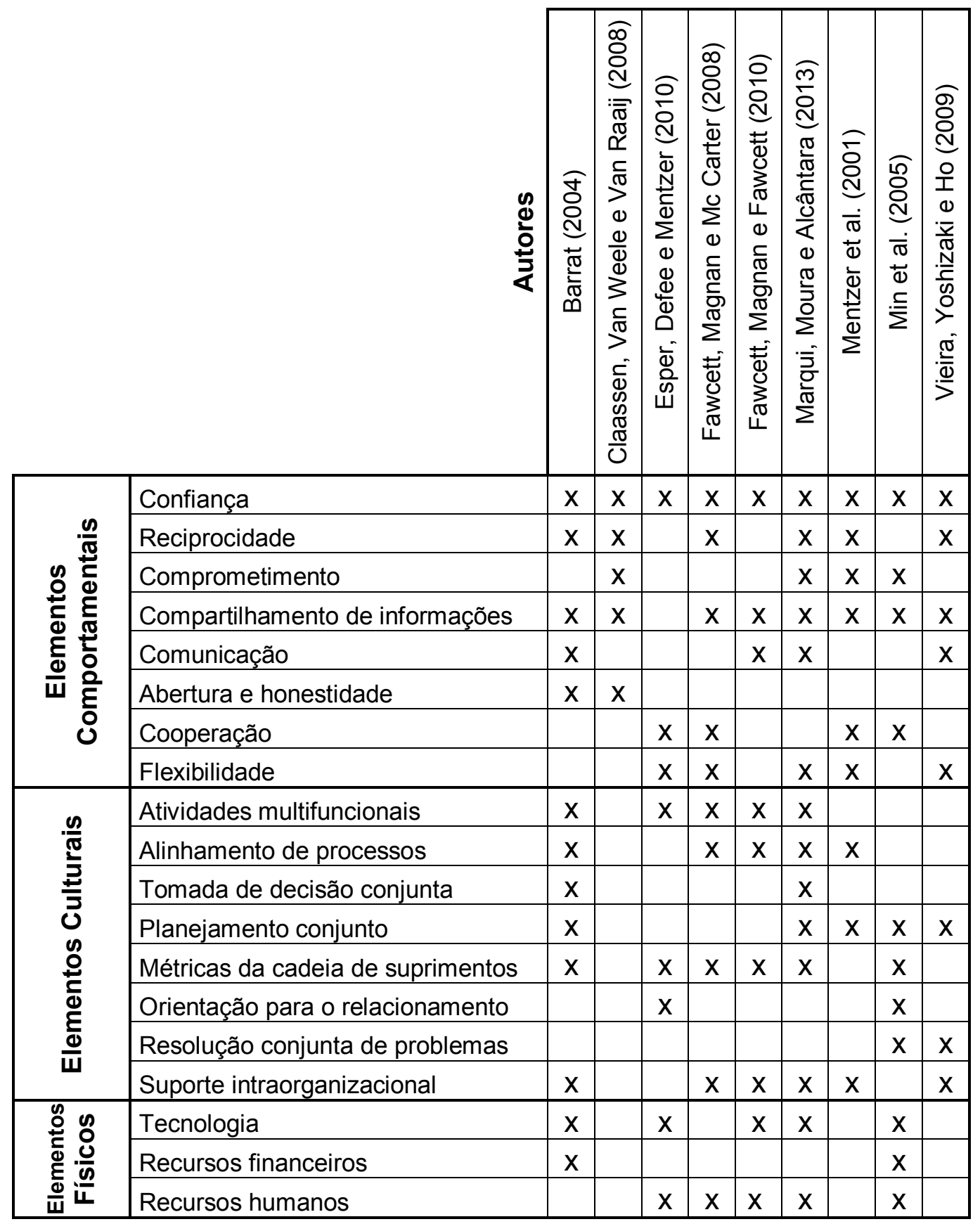

Nota: $O$ ' $X$ ' indica que o elemento é mencionado na literatura consultada.

Fonte: Elaborada pelos autores (2013)

Os elementos comportamentais estão relacionados à disposição das empresas em colaborar na cadeia de suprimentos. A confiança é a base para qualquer relacionamento colaborativo (BARRAT, 2004; MARQUI; MOURA; ALCÂNTARA, 2013; MIN et al., 2005; VIEIRA; YOSHIZAKI; HO, 2009). Através dela,

Revista Produção Online, Florianópolis, SC, v.14, n. 3, p. 1024-1049, jul./set. 2014. 
as empresas compartilham crenças convergentes, permitindo a colaboração. Para Mentzer et al. (2001), confiança representa a vontade de contar com um parceiro em quem se tem segurança e é importante para superar as dificuldades mútuas, relacionadas ao poder, conflito, menor rentabilidade. A reciprocidade (ou interdependência) está relacionada à vontade de compartilhar riscos e recompensas (BARRAT, 2004; FAWCETT; MAGNAN; MC CARTER, 2008; MARQUI; MOURA; ALCÂNTARA, 2013; MENTZER et al., 2001; VIEIRA; YOSHIZAKI; HO, 2009). Para Mentzer et al. (2001), refere-se à necessidade de manter um relacionamento para atingir os objetivos e promove a vontade de compartilhar informações e realizar planejamento conjuntamente. O comprometimento garante a continuidade do relacionamento a longo prazo (CLAASEN; VAN WEELE; VAN RAAIJ, 2008; MENTZER et al., 2001). Para Min et al. (2005), o comprometimento também está relacionado com a tolerância perante às dificuldades e deficiências do parceiro. $O$ compartilhamento de informações é importante para criar entendimentos comuns em relação a metas e objetivos (CLAASEN; VAN WEELE; VAN RAAIJ, 2008) e é fundamental para garantir as ações conjuntas (VIEIRA; YOSHIZAKI; HO, 2009). Barrat (2004), Fawcett, Magnan e Mc Carter (2008) comentam sobre a importância da transparência e qualidade dos fluxos de informação, garantindo que a informação esteja acessível no lugar correto, no tempo correto e nas mãos das pessoas certas. A comunicação mantém o compartilhamento de informações e cria um entendimento comum compartilhado (BARRAT, 2004) e auxilia no entendimento das dificuldades e estratégias do parceiro (VIEIRA; YOSHIZAKI; HO, 2009). Marqui, Moura e Alcântara (2013) falam que a comunicação antecipada de potenciais problemas é recomendada para o desenvolvimento de relacionamentos mais próximos. Semelhantemente, Barrat (2004) mostra que a abertura e honestidade envolve informar o parceiro sobre eventuais problemas, antes que eles aconteçam, permitindo a criação de planos de contingência. A cooperação limita os comportamentos oportunistas, com pensamento sistêmico na parceria da cadeia de suprimentos, e não por meio de objetivos individuais (MENTZER et al., 2001). Por fim, a flexibilidade está relacionada à capacidade de reação às mudanças do ambiente e do mercado (ESPER; DEFEE; MENTZER, 2010; MARQUI; MOURA; ALCÂNTARA, 2013; MENTZER, 2001; VIEIRA; YOSHIZAKI; HO, 2009). 
Neste sentido, Kemczinski et al. (2007) desenvolve um trabalho a fim de descobrir a pertinência, concorrência e complementaridade do tema colaboração e cooperação e acaba por afirmar que estes são termos complementares. Este trabalho adota também essa visão, mostrando que a cooperação envolve o trabalho conjunto entre os membros da cadeia de suprimentos que contribui para o aumento da colaboração, entendido aqui como um esforço coordenado na busca de objetivos comuns.

Os elementos culturais estão diretamente relacionados à cultura organizacional, influenciando a implantação e manutenção das práticas colaborativas. É importante que as empresas envolvidas na prática colaborativa livrem-se da estrutura funcional e orientem-se a partir de atividades multifuncionais (ESPER; DEFEE; MENTZER, 2010; FAWCETT; MAGNAN; MC CARTER, 2008; MARQUI; MOURA; ALCÂNTARA, 2013), uma vez que, segundo Barrat (2004), as fronteiras internas à organização e entre as empresas restringem o fluxo de informação e minimiza a confiança entre os parceiros. Mentzer et al. (2001) comenta sobre a importância da integração dos processos chave entre as empresas, evitando os retrabalhos e garantindo a eficiência dos processos. Barrat (2004) fala sobre a importância da tomada de decisão conjunta, principalmente no que diz respeito à previsão e Marqui, Moura e Alcântara (2013) apontam que decisões táticas, tais como segmentação de mercado, variedade de produtos, nível de serviço ao cliente, promoções e previsões; bem como as decisões operacionais (atendimento das ordens) devem ser realizadas em conjunto. É extremamente importante que haja o planejamento das metas e objetivos da parceria (BARRAT, 2004; MENTZER et al., 2001; MIN et al., 2005; MOURA; MARQUI; ALCÂNTARA, 2013). E Min et al. (2005) adicionam que os parceiros devem trabalhar conjuntamente para resolver os problemas da cadeia de suprimentos. O desenvolvimento de métricas que contemplem a cadeia de suprimentos como um todo é outro fator muito importante (ESPER; DEFEE; MENTZER, 2010; FAWCETT; MAGNAN; MC CARTER, 2008), garantindo que todos na cadeia de suprimentos operem na mesma direção, com alinhamento de metas e objetivos (BARRAT, 2004). Min et al. (2005) acrescenta ainda que o sucesso dos esforços colaborativos não podem ser garantidos se o desempenho não for corretamente monitorado e medido. Esper, Defee e Mentzer 
(2010) explicam que um antecedente para a colaboração é a orientação para a cadeia de suprimentos, com o comprometimento da alta gerência fornecendo o suporte intraorganizacional necessário, uma vez que ela é responsável por modelar os valores, orientação e direção da organização (MENTZER et al., 2001).

Finalmente, os elementos físicos estão relacionados com a tecnologia e os recursos necessários para viabilizar a implantação da prática do VMI. Barrat (2004) e Min et al. (2005) sugerem que os participantes da colaboração devem estar preparados para comprometer recursos financeiros e não-financeiros tanto nos estágios iniciais, quanto nos estágios avançados da colaboração, na forma de tempo, dinheiro, treinamento, atualização de tecnologias, etc. Barrat (2004) discute que a colaboração não precisa se basear na tecnologia, e que, em estágios iniciais, tecnologias simples, como o e-mail, podem ser efetivos para a colaboração; no entanto, com o aumento do volume de dados, a tecnologia se torna importante e pode viabilizar o acesso aos dados em tempo real. Fawcett, Magnan e Fawcett (2010) compartilham da mesma ideia, mostrando que anteriormente, tecnologia inadequada era vista como uma barreira para as iniciativas de colaboração, porém, os investimentos maciços em tecnologia nos últimos anos não trouxeram avanços significativos nos relacionamentos. Em relação aos recursos humanos, Esper, Defee e Mentzer (2010) sugerem que as empresas devem se preocupar com a contratação e motivação dos funcionários com conhecimentos e habilidades específicas em logística e cadeia de suprimentos; enquanto Fawcett, Magnan e Mc Carter (2008) falam sobre a importância de dar poder de decisão às pessoas e Fawcett, Magnan e Fawcett (2010) apontam que as empresas estão buscando melhorar suas habilidades de formação de equipes.

\section{METODOLOGIA}

Baseada na classificação dos tipos de pesquisa proposto por Collis e Hussey (2005) quanto ao objetivo, processo, lógica e resultado da pesquisa, o presente estudo constitui-se um estudo exploratório, qualitativo, dedutivo e aplicado, respectivamente. Trata-se de uma pesquisa exploratória, uma vez que busca identificar as barreiras que limitam os ganhos potenciais da prática do VMI e também identificar os fatores considerados críticos para o sucesso da prática. $O$ caráter 
qualitativo deste estudo permite capturar toda a complexidade do fenômeno em seu ambiente natural (MARTINS, 2012), dando mais flexibilidade à pesquisa. Ensslin e Vianna (2008) complementam ainda que a utilização de métodos qualitativos na Engenharia de Produção permite considerar a dinâmica entre o mundo real e os atores, através da interpretação, compreensão e atribuição de significado aos fenômenos. Em relação à lógica, este estudo é considerado dedutivo no qual, segundo Collis e Hussey (2005), foi desenvolvida uma estrutura conceitual e teórica, que foi, posteriormente, testada empiricamente. E finalmente, é classificada como aplicada, uma vez que busca gerar conhecimentos para a aplicação do VMI nas empresas.

Com relação ao método, o estudo de caso mostrou ser o mais adequado às necessidades desta pesquisa que, conforme Yin (2005, p.32), "é uma investigação empírica que investiga um fenômeno contemporâneo dentro de seu contexto da vida real, especialmente quando os limites entre o fenômeno e o contexto não estão claramente definidos". Gil (2002, p.54) afirma que o estudo de caso consiste no "estudo profundo e exaustivo de um ou poucos objetos, de maneira que permita seu amplo e detalhado conhecimento (...)". Coerentemente, Johnson, Godsell e Ke (2013) afirmam que o estudo de caso é adequado para campos ainda imaturos, tais como Gestão de Operações e Gestão da Cadeia de Suprimentos, que ainda carecem de definições consensuais.

A prática do VMI em questão foi analisada através de um estudo de caso em uma empresa multinacional de grande porte, fornecedora de bens de consumo não duráveis, instalada no estado de São Paulo. A escolha desta empresa deu-se pela reconhecida importância da matriz norte-americana no desenvolvimento e uso da prática do VMI, em função de ter sido uma das pioneiras na sua adoção. Foi utilizado o estudo de caso único, por se tratar de um caso representativo ou típico, no qual, segundo Yin (2005), as informações sobre as experiências da instituição resultam em aprendizado importante.

Como forma de coleta de dados foi utilizada técnicas de documentação direta, por meio de pesquisas e consultas em minutas de reuniões, relatórios e registros em arquivos; documentação indireta através da pesquisa de campo; e observação direta intensiva por meio de observação e entrevista em profundidade. 
As entrevistas foram realizadas com o gerente de serviço ao cliente e o supervisor de serviço ao cliente durante o segundo semestre de 2011, a partir de um roteiro semiestruturado (que passou por um pré-teste) e com retorno aos entrevistados para melhor entendimento de alguns pontos não esclarecidos durante a entrevista.

Como parte do protocolo de pesquisa para se chegar aos entrevistados, a conveniência se deu por ser um caso anteriormente conhecido dos pesquisadores, portanto, com contatos estabelecidos antes da pesquisa.

Para a análise dos dados utilizou-se a progressão analítica que, segundo Miles e Huberman (1994), é a progressão da descrição para a explicação. Nessa direção, os autores sugerem que a progressão analítica comece contando uma história a fim de construir um "mapa" na intenção de formalizar os elementos da história, localizando as variáveis-chave. O próximo passo envolve a construção de uma teoria ou modelo, ou seja, ligação entre as variáveis e forma como influenciam umas às outras.

\section{ANÁLISE DA UTILIZAÇÃO DO VMI NA EMPRESA: ESTUDO DE CASO}

A empresa estudada está entre as maiores empresas de bens de consumo do mundo. Com sede nos EUA, está presente no Brasil há mais de vinte anos com cerca de 30 marcas em diferentes categorias de produtos e vem apresentando altas taxas de crescimento se comparada às outras unidades ao redor do mundo. Dentre seus clientes, estão as grandes redes varejistas e atacadistas presentes no Brasil e as principais redes de drogarias.

O sistema VMI analisado é um projeto piloto para a empresa, iniciado no final do ano de 2010, por iniciativas do cliente varejista, com alto comprometimento da alta gerência de ambas as empresas devido aos bons resultados obtidos com a adoção da prática pelas mesmas empresas em outros países e também devido às pressões exercidas pelas matrizes.

O cliente em questão é uma das maiores empresas do setor varejista do mundo. No Brasil, está presente em estados das regiões nordeste, centro-oeste, sudeste e sul, e possui diversos formatos de loja: hipermercados, supermercados, clubes de compras, lojas de atacado e de vizinhança.

Revista Produção Online, Florianópolis, SC, v.14, n. 3, p. 1024-1049, jul./set. 2014. 
O fornecedor dedica um time de pessoas multifuncionais para atender exclusivamente o cliente em questão, uma vez que este é um cliente-chave e extremamente importante para o negócio. Este time é composto por recursos das áreas de logística, vendas, finanças, marketing, tecnologia da informação e inteligência de mercado.

A figura 2 apresenta as principais barreiras que limitam os potenciais resultados da prática do VMI na empresa estudada, bem como os fatores considerados críticos para o sucesso da mesma, de acordo com os elementos comportamentais, culturais e físicos citados anteriormente (figura 1).

Figura 2 - Barreiras e Fatores Críticos de Sucesso do processo VMI estudado

\begin{tabular}{|c|c|c|}
\hline & Barreiras & Fatores Críticos de Sucesso \\
\hline 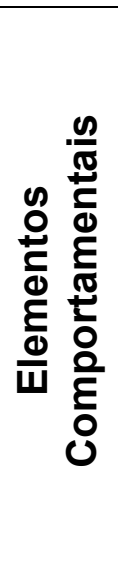 & $\begin{array}{l}\text { - Baixo nível de confiança devido às } \\
\text { restrições de capacidade produtiva e } \\
\text { capacidade de recebimento dos CD's. }\end{array}$ & $\begin{array}{l}\text { - Presença do funcionário do fornecedor } \\
\text { no escritório administrativo do cliente e } \\
\text { reuniões quinzenais, aumentando a } \\
\text { comunicação, cooperação, abertura e } \\
\text { honestidade entre as empresas; } \\
\text { - Compartilhamento das restrições } \\
\text { produtivas por parte do fornecedor e } \\
\text { calendário de eventos por parte do } \\
\text { cliente, aumentando a reciprocidade e } \\
\text { comprometimento; } \\
\text { - Alteração na estrutura das equipes } \\
\text { multifuncionais do fornecedor resultou em } \\
\text { maior flexibilidade para atender as } \\
\text { necessidades do mercado. }\end{array}$ \\
\hline 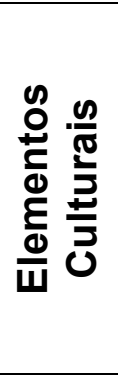 & $\begin{array}{l}\text { - Atividades funcionais; } \\
\text { - Processos ainda não muito estruturados } \\
\text { entre as empresas; } \\
\text { - Métricas funcionais e internas. }\end{array}$ & $\begin{array}{l}\text { - Equipes multifuncionais; } \\
\text { - Processo sendo alinhado entre as } \\
\text { empresas; } \\
\text { - Evolução das atividades de } \\
\text { planejamento, tomada de decisão e } \\
\text { resolução de problemas realizados } \\
\text { conjuntamente pelas empresas; } \\
\text { - Suporte da alta gerência de ambas as } \\
\text { empresas. }\end{array}$ \\
\hline 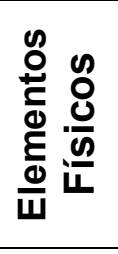 & $\begin{array}{l}\text { - Falta de integração dos sistemas de } \\
\text { informação entre as empresas. }\end{array}$ & $\begin{array}{l}\text { - Fornecedor com acesso ao sistema do } \\
\text { cliente; } \\
\text { - Recursos financeiros disponíveis para } \\
\text { melhoria da prática; } \\
\text { - Funcionários do fornecedor dedicados ao } \\
\text { cliente. }\end{array}$ \\
\hline
\end{tabular}

Fonte: Adaptado de Freitas,Tomas, Alcântara (2013)

O cenário atual mostra que a capacidade produtiva do fornecedor é menor que sua demanda e, além disto, há restrições de volume de recebimento de Revista Produção Online, Florianópolis, SC, v.14, n. 3, p. 1024-1049, jul./set. 2014. 
produtos tanto no centro de distribuição quanto nas lojas do cliente. Tal cenário faz com que a confiança entre os agentes fique abalada.

Como forma de minimizar o impacto da falta de confiança, são realizadas reuniões constantes (quinzenais) e há a permanência do supervisor de serviço ao cliente no escritório do varejista alguns dias da semana, o que permite maior agilidade na identificação e solução de problemas e aumenta a comunicação e troca de informações entre os parceiros. Com isso, o fornecedor começou a compartilhar com o cliente seus principais problemas, tais como problemas com matérias-primas e limites de produção mensal. Por sua vez, o varejista começou a compartilhar seu calendário de eventos, permitindo o planejamento conjunto dos eventos, através da realização da previsão conjunta da demanda e também desenvolvimento de produtos exclusivos para cada evento. Isto aumenta a abertura e honestidade entre as empresas, contribuindo para a formação de um relacionamento sólido, com confiança crescente no parceiro comercial e comprometimento com o relacionamento, buscando soluções recíprocas e aumentando a venda de ambas as empresas.

O trabalho conjunto promove maior cooperação entre os atores, que buscam sempre se flexibilizar as necessidades do mercado. Um exemplo recente de flexibilidade para melhorar o relacionamento e comunicação entre as duas empresas foi a reestruturação das atividades entre os supervisores de serviço ao cliente, que antes lidavam, cada um, com uma bandeira de atacado e uma (ou duas) bandeira(s) de varejo e atualmente estão responsáveis somente pelas bandeiras de varejo ou somente pelas bandeiras de atacado.

As principais barreiras do processo VMI entre as empresas estão relacionadas aos elementos culturais. Embora o fornecedor trabalhe com times multifuncionais com foco nos clientes, as métricas ainda são funcionais e não estão interligadas com o cliente. Neste sentido, a equipe comercial do fornecedor possui metas de vendas mensais, enquanto uma das principais medidas de desempenho do departamento de logística do cliente é dada pelo nível de estoque no último dia de cada mês. Com isso, os funcionários da empresa varejista fazem pedidos de grande quantidade na última semana do mês (impulsionados pela equipe comercial do fornecedor que precisa atingir sua meta), que será entregue somente na primeira 
semana do próximo mês. Assim, o varejista mantém altos níveis de inventário nos primeiros dias do mês, que serão reduzidos até o final do mês, quando haverá nova medição.

Esses comportamentos incorrem em custos adicionais para toda a cadeia de suprimentos, com o aumento do custo com inventário e os custos dos caminhões extras que serão necessários para atender ao pico de entregas no final do mês. Além disto, consoante com o trabalho de Mulato e Oliveira (2006), essas políticas comerciais das empresas contribuem para o congestionamento das docas do cliente, resultando em transtornos logísticos e reduzindo a rentabilidade.

Conforme já mencionado, os aspectos colaborativos entre as empresas vêm evoluindo, com novos processos sendo alinhados, decisões sendo tomadas conjuntamente e também através do planejamento conjunto, devido principalmente ao trabalho realizado pelo supervisor de serviço ao cliente dentro das instalações do cliente varejista.

A orientação para o relacionamento já faz parte da cultura da empresa, uma vez que há a divisão multifuncional desenvolvida especialmente para atender as necessidades do cliente. Há todo um suporte intraorganizacional para este cliente, visto sua importância para a companhia (segundo maior volume de vendas) e também devido ao altíssimo grau de cumplicidade entre as duas empresas nos Estados Unidos. Neste sentido, existe um grupo internacional do fornecedor, para a rede varejista em questão, especialmente focado no relacionamento entre essas duas empresas, que visitam o Brasil a cada três meses em busca de oportunidades de melhoria e também para auxiliar no relacionamento entre as unidades brasileiras, através de exemplos bem sucedidos ao redor do mundo.

Em relação aos elementos físicos que influenciam no $\mathrm{VMI}$, a tecnologia adotada pelas empresas é satisfatória para o monitoramento do inventário das lojas do cliente e também para o processo de previsão de demanda. O fornecedor tem acesso online ao sistema do cliente, que possui informações consistentes e com apenas um dia de atraso. Com esse sistema, o fornecedor pode montar relatórios customizados com as informações que julgue necessário, podendo chegar ao detalhamento ao nível de item por loja do dia anterior. Uma deficiência, porém, 
advém da falta de integração dos sistemas de informação entre as empresas, o que gera duplicação de trabalhos e uma variedade de processos não automatizados.

A existência do grupo internacional facilita a disponibilidade de recursos financeiros e humanos e também incentiva novas práticas que melhorem 0 relacionamento entre as empresas através do apoio financeiro. O suporte internacional também permite que o time possua mais funcionários dedicados ao cliente em questão, quando comparados aos times da mesma empresa dedicados a outros clientes importantes.

Uma informação interessante obtidas com as entrevistas mostra que as empresas estudadas ainda não alcançaram o mais avançado nível do VMI, no qual a responsabilidade de gerenciar o estoque e o processo de reposição passa para o fornecedor. Tal fato deve-se à aplicação recente da prática e também à prematura confiança entre os agentes. Nesta direção, o cliente possui um sistema de reposição automática de estoque a partir de parâmetros mínimo e máximo. Com a adoção do VMI, foi dada ao fornecedor a responsabilidade de administrar esses parâmetros, sob as expectativas do cliente de que o inventário gire dentro do prazo de pagamento e que não faltem produtos nas lojas.

Alguns produtos necessitam intervenção manual de colocação de pedidos quando ocorre um evento, por exemplo. O supervisor de serviço ao cliente fica responsável pela tomada de decisão de quantidade extra a ser pedida e alinhamento das expectativas de datas de entrega, juntamente com o cliente, já com a visibilidade de capacidade de produção/entrega dos pedidos.

\section{CONSIDERAÇÕES}

A revisão da literatura possibilitou a identificação dos elementos que influenciam a implantação e manutenção da prática do VMI, e norteou o estudo de caso, que identificou, na empresa estudada, as barreiras que limitam o potencial do VMI, bem como os fatores críticos de sucesso da parceria.

Dentre os elementos comportamentais, a restrição de capacidade produtiva do fornecedor, aliada à restrição de capacidade de recebimento do cliente são responsáveis pelo baixo nível de confiança entre os agentes, agindo como uma barreira no relacionamento entre eles. Para contornar a situação, as empresas então

Revista Produção Online, Florianópolis, SC, v.14, n. 3, p. 1024-1049, jul./set. 2014. 
buscam o relacionamento aberto, a comunicação, a cooperação, a flexibilidade, a troca de informação, abertura e honestidade para desfrutarem dos benefícios do VMI. Coerentemente com Barrat (2004), a abertura e honestidade entre as empresas podem desenvolver a confiança, respeito e comprometimento, como resultado da melhoria da segurança e confiabilidade.

Apesar da existência de barreiras funcionais em ambas as empresas, evidenciadas pelas métricas departamentais, o relacionamento entre as empresas caminham para a colaboração, já com suporte intraorganizacional, planejamento e tomada de decisão realizados conjuntamente, mostrando um grande avanço entre os elementos culturais.

Os elementos físicos já estão bem desenvolvidos entre as empresas, que dispõem de recursos financeiros, humanos e tecnológicos suficientes para que os ganhos ao longo da cadeia de suprimentos sejam percebidos.

É interessante notar que, coerentemente as proposições de Whipple e Russel (2007), o VMI representa o primeiro nível de colaboração e é a base para o estabelecimento dos outros níveis mais avançados de colaboração. A análise do caso mostra claramente que a colaboração entre as empresas estudadas está na fase de transição entre os estágios de gestão de transações colaborativas e gestão de eventos colaborativos. Uma vez resolvido os problemas de reposição de estoques, a partir da visibilidade da demanda do consumidor final, as empresas começam a perceber que eventos como promoções, lançamentos de novos produtos também interferem diretamente nos níveis de estoque das lojas e, com isso, começam a trocar informações e planejar conjuntamente os eventos (calendário, demanda e elaboração de produtos promocionais).

De maneira análoga, é possível perceber que as empresas estão também na transação entre a comunicação e a colaboração limitada proposta por Danese (2007), com as empresas evoluindo da simples troca de dados e informações para sincronização de planos e gerenciamento das exceções. O exemplo claro neste caso é o planejamento conjunto dos eventos, no qual o fornecedor e o cliente analisam conjuntamente a capacidade de produção/entrega dos pedidos e alinham com as expectativas de data de entrega. 
Adicionalmente, de acordo com o trabalho de Marqui, Moura e Alcântara (2013), é possível verificar aspectos da cooperação e da coordenação no relacionamento das empresas, mas elas ainda não conseguiram alcançar o último degrau da colaboração. A comunicação, transparência e interdependência através do compartilhamento das informações (acesso online ao sistema do cliente) podem ser percebidas pelo estudo de caso. As características de flexibilidade e sincronização da tomada de decisão do estágio de coordenação também podem ser notadas principalmente em itens promocionais ou eventos específicos. A liderança, porém, aparentemente não é exercida por nenhuma empresa individualmente. Dentre os comportamentos da coordenação, o planejamento conjunto e o envolvimento da alta gerência estão presentes no relacionamento estudado. As empresas, porém, ainda não investiram em TI e nem na documentação e padronização dos processos. As características do último degrau que estão sendo construídas, a partir do relato do caso, são a confiança e o comprometimento.

Dos resultados obtidos a partir do estudo de caso e da revisão da literatura, as seguintes proposições são apresentadas:

P1: A adoção de práticas colaborativas pelas empresas ocorre através de um processo evolutivo, iniciando-se com a adoção de práticas mais simples;

P2: À medida que as barreiras, compostas de elementos comportamentais, culturais e físicos são ultrapassadas, práticas mais complexas são implantadas;

P3: À medida que os fatores críticos, também relacionados aos elementos comportamentais, culturais e físicos, são incorporados pelas empresas, práticas mais complexas são implantadas.

A primeira proposição está relacionada aos trabalhos de Whipple e Russel (2007), Danese (2007) e Marqui, Moura e Alcântara (2013), que propõem estágios de evolução do relacionamento colaborativo. A proposição deste trabalho, porém, é que as práticas de colaboração na cadeia de suprimentos também são implantadas pelas empresas a partir de um processo evolutivo, no qual práticas mais simples são adotadas e evoluem para práticas mais complexas.

As duas últimas proposições relacionam-se com o trabalho de Stamato Neto e Alcântara (2013): à medida que as empresas percebem uma barreira que limita seus ganhos potenciais, ela adquire novos recursos (tangíveis ou intangíveis) que são 
organizados para se tornarem uma capacitação. No caso da pesquisa em questão, consideram-se como recursos os elementos culturais, comportamentais e físicos. $E$ também estão relacionadas com o trabalho de Fawcett, Magnan e Fawcett (2010), que argumenta que as empresas devem colaborar entre si, compartilhando recursos e capacitações complementares para criar valor ao cliente, criando fontes de vantagem competitiva. Estes últimos autores também se referem às barreiras ou resistências à colaboração e aos facilitadores da colaboração, aqui entendidos como os fatores críticos de sucesso.

Este artigo contribui para o entendimento dos elementos que influenciam o resultado esperado da prática do VMI, o que pode auxiliar os gestores das empresas usuárias ou interessadas em aplicar a prática. Neste sentido, os gestores podem identificar aspectos internos às suas empresas que podem limitar o potencial da prática do VMI e criar planos de ação para corrigir os problemas internos. Além disto, podem identificar os elementos que contribuem efetivamente para o sucesso da prática e fortalecê-los internamente. O estudo de caso também aponta algumas alternativas criadas pela empresa estudada para contornar as deficiências existentes em relação aos fatores comportamentais e culturais e melhorar o relacionamento com seu cliente, que podem ser adaptados para a realidade de outras empresas que utilizam ou pretendem adotar o VMI.

As proposições apresentadas abrem espaço para novas pesquisas na área de gestão de cadeia de suprimento, centradas na diferentes iniciativas e práticas de colaboração adotadas.

Finalmente, uma limitação deste trabalho é a realização do estudo unicamente sob a ótica do fornecedor, e em apenas uma cadeia de suprimentos. Pesquisas futuras podem avaliar a adoção de iniciativas de colaboração da cadeia de suprimentos em outras empresas e em outros segmentos de mercados. Além disto, outra limitação é decorrente da falta de estudos empíricos com o mesmo foco de análise desta pesquisa, o que impossibilita a discussão sobre os possíveis modos de implantação do VMI, como também inibe a possibilidade de comparação dos resultados obtidos.

Nota

Texto inédito, financiado pelo CNPq, parte da pesquisa de mestrado em andamento.

Revista Produção Online, Florianópolis, SC, v.14, n. 3, p. 1024-1049, jul./set. 2014. 


\section{REFERÊNCIAS}

ATTARAN, M.; ATTARAN, S. Collaborative supply chain management: The most promising practice for building efficient and sustainable supply chains. Business Process Management Journal, v. 13, n. 3, p. 390-404, 2007. http://dx.doi.org/10.1108/14637150710752308

BALLOU, R. H. The evolution and future of logistics and supply chain management. European Business Review, v. 19, n. 4, p. 332-348, 2007. http://dx.doi.org/10.1108/09555340710760152

BARRAT, M. Understanding the meaning of collaboration in the supply chain. Supply Chain Management: An International Journal, v. 9, n. 1, p. 30-42, 2004. http://dx.doi.org/10.1108/13598540410517566

BLATERWICK, A. Vendor-managed inventory: fashion fad or important supply chain strategy? Supply Chain Management, v. 3, n. 1, p. 10-11, 1998.

http://dx.doi.org/10.1108/13598549810200825

CLAASSEN, M. J. T.; VAN WEELE, A. J.; VAN RAAIJ, E. M. Performance outcomes and success factors of vendor managed inventory (VMI). Supply Chain

Management: An International Journal, v.13, n.6, p. 406-414, 2008. http://dx.doi.org/10.1108/13598540810905660

COLLIS, J.; HUSSEY, R. Pesquisa em Administração: Um guia prático para alunos de graduação e pós-graduação. $2^{a}$ ed. Porto Alegre: Bookmamn, 2005.

DANESE, P. The extended VMI for coordinating the whole supply chain network. Journal of Manufacturing Technology Management, v. 17, n. 7, p. 888-907, 2006. http://dx.doi.org/10.1108/17410380610688223

DANESE, P. Designing CPFR collaborations: insights from seven case studies. International Journal of Operations \& Production Management, v. 27, n. 2, p. 181-204, 2007. http://dx.doi.org/10.1108/01443570710720612

DAUGHERTY, P. J.; MYERS, M. B.; AUTRY, C. W. Automatic Replenishment Programs: an empirical examination. Journal of Business Logistics, v. 20, n. 2, p. 63-82, 1999.

DISNEY, S. M; TOWILL, D. R. Vendor-managed inventory and bullwhip reduction in a two-level supply chain. International Journal of Operations \& Production Management, v. 23, n. 6, p. 625-651, 2003. http://dx.doi.org/10.1108/01443570310476654

ELVANDER, M. S.; SARPOLA, S.; MATTSON, S-A. Framework for characterizing the design of VMI systems. International Journal of Physical Distribution \& Logistics Management, v. 37, n. 10, p. 782-798, 2007.

http://dx.doi.org/10.1108/09600030710848914 
ENSSLIN, L.; VIANNA, W. B. O design na pesquisa quali-quantitativa em engenharia de produção: questões epistemológicas. Revista Produção Online, v. 8, n. 1, 2008.

ESPER, T. L.; DEFEE, C. C.; MENTZER, J. T. A framework of supply chain orientation. The International Journal of Logistics Management, v. 21, n. 2, p. 161-179, 2010. http://dx.doi.org/10.1108/09574091011071906

FAWCETT, S. E.; MAGNAN, G. M.; FAWCETT, A. M. Mitigating resisting forces to achieve the collaboration-enabled supply chain. Benchmarking: An International Journal, v. 17, n. 2, p. 269-293, 2010.

FAWCETT, S. E.; MAGNAN, G. M.; MCCARTER, M. W. Benefits, barriers, and bridges to effective supply chain management. Supply Chain Management: An International Journal, v. 13, n. 1, p. 35-48, 2008.

http://dx.doi.org/10.1108/13598540810850300

FERREIRA, H. S. R.; ALCANTARA, R. L. C. Mecanismos de coordenação no canal de distribuição automotivo: algumas contribuições. Revista Produção Online. v. 11, n. 3, p. 619-647, 2011. http://dx.doi.org/10.14488/1676-1901.v11i3.461

FREITAS, D.C.; TOMAS, R. N.; ALCÂNTARA, R. L. C. Estoque gerenciado pelo fornecedor (VMI): análise das barreiras e fatores críticos de sucesso em empresas de grande porte. Revista de Administração da UNIMEP, v. 11, n. 3, Setembro/Dezembro, p. 221-252, 2013.

FUGATE, B.; SAHIN, F.; MENTZER, J. T. Supply Chain Management Coordination Mechanisms. Journal of Business Logistics, v. 27, n. 2, p. 129-161, 2006. http://dx.doi.org/10.1002/j.2158-1592.2006.tb00220.x

GIL, A. C. Como elaborar projetos de pesquisa. 4. ed. São Paulo: Atlas, 2002.

JOHNSON, M.; GODSELL, J.; KE, Q. What does good look like? Identifying quality criteria for case studies in operations and supply chain management. In: $\mathbf{2 0}^{\text {th }}$ International Annual EurOMA Conference Operations Management at the Heart of the Recovery, 2013, Dublin. 20 ${ }^{\text {th }}$ International Annual EurOMA Conference Proceedings, 2013.

KEMCZINSKI, A. et al. Colaboração e cooperação: pertinência, concorrência ou complementaridade. Revista Produção Online, v. 7, n. 3, 2007.

LAMBERT, D. M. Supply Chain Management. In: (Editor) Supply Chain Management: Processes, Partnerships, Performance. 3. ed. Florida: Supply Chain Management Institute, 2008. Capítulo 1, p. 1-23.

MARQUI, A. C.; MOURA, K. S.; ALCÂNTARA, R. L. C. Collaborative supply chain : a conceptual model for operationalisation. International Journal of Management and Decision Making, v. 12, n. 3, p. 195-214, 2013.

http://dx.doi.org/10.1504/IJMDM.2013.056458 
MARTINS, R. A. Abordagens quantitativa e qualitativa. In: MIGUEL, P. A. C. (Coordenador) Metodologia de pesquisa em engenharia de produção e gestão de operações. 2. ed. Rio de Janeiro: Elsevier: ABEPRO, 2012. Capítulo 3, p. 47-63.

MENTZER, J. T. et al. Defining supply chain management. Journal of Business Logistics, v. 22, n. 2, 2001. http://dx.doi.org/10.1002/j.2158-1592.2001.tb00001.x

MILES, M. B.; HUBERMAN, A. M. Qualitative data analysis: an expanded sourcebook. 2. ed. London: Sage Publications, 1994. 338 p.

MIN S. et al. Supply chain collaboration: What's happening? The International Journal of Logistics Management, v. 16, n. 2, p. 237-256, 2005.

http://dx.doi.org/10.1108/09574090510634539

MULATO, F. M.; OLIVEIRA, M. M. B. O impacto de um sistema de agendamento antecipado de docas para carga e descarga na gestão da cadeia de suprimentos. Revista Produção Online. v. 6, n. 3, 2006.

PIRES, S. R. I. Gestão da cadeia de suprimentos: conceitos, estratégias, práticas e casos. 2. ed. São Paulo: Atlas, 2010.

REDDY, M. VRAT, P. Vendor managed inventory model: a case study. Journal of Advances in Management Research, v. 4, n. 1, p. 83-88, 2007.

http://dx.doi.org/10.1108/97279810780001254

SARI, K. Exploring the benefits of vendor managed inventory. International Journal of Physical Distribution \& Logistics Management, v. 37, n. 7, p. 529-545, 2007. http://dx.doi.org/10.1108/09600030710776464

SARI, K. Inventory inaccuracy and performance of collaborative supply chain practices. Industrial Management \& Data Systems, v. 108, n. 4, p. 495-509, 2008. http://dx.doi.org/10.1108/02635570810868353

SOOSAY, C. A; HYLAND, P. W.; FERRER M. Supply chain collaboration: capabilities for continuous innovation. Supply Chain Management: An International Journal, v. 13, n. 2, p.160-169, 2008.

http://dx.doi.org/10.1108/13598540810860994

STAMATO NETO, J.; ALCÂNTARA, R. L. C. Competências essenciais presentes em frigoríficos brasileiros exportadores para a Europa: um estudo multicaso. Revista Produção Online, v. 13, n. 1, p. 180-207, jan./mar. 2013.

http://dx.doi.org/10.14488/1676-1901.v13i1.1107

VIEIRA, J.; YOSHIZAKI, H.; HO, L. Collaboration intensity in the Brazilian supermarket retail chain. Supply Chain Management: An International Journal, v.14, n.1, p. 11-21, 2009. http://dx.doi.org/10.1108/13598540910927269

VIGTIL, A. Information exchange in vendor managed inventory. International Journal of Physical Distribution \& Logistics Management, v. 37, n. 2, p. 131-147, 2007. http://dx.doi.org/10.1108/09600030710734848

Revista Produção Online, Florianópolis, SC, v.14, n. 3, p. 1024-1049, jul./set. 2014. 
WALLER, M.; JOHNSON, M. E.; DAVIS, T. Vendor-managed inventory in the retail supply chain. Journal of Business Logistics, v. 20, n. 1, p. 183-203, 1999.

WHIPPLE, J. M.; RUSSEL, D. Building supply chain collaboration: a typology of collaborative approaches. The International Journal of Logistics Management, $v$. 18, n. 2, p. 174-196, 2007. http://dx.doi.org/10.1108/09574090710816922

WILLIAMS, B. D.; TOKAR, T. A review of inventory management research in major logistics journals: Themes and future directions. The International Journal of Logistics Management, v. 19, n. 2, p. 212-232, 2008.

http://dx.doi.org/10.1108/09574090810895960

YIN, R. K. Estudo de caso: planejamento e métodos. 3. ed. Porto Alegre: Bookman, 2005.

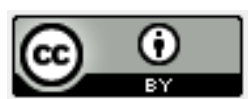

Artigo recebido em 14/08/2013 e aceito para publicação em 14/04/2014 DOI: http://dx.doi.org/ 10.14488/1676-1901.v14i3.1654 